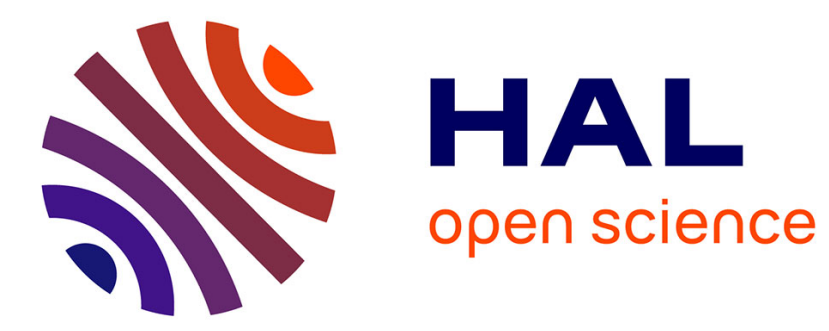

\title{
Pleasantness of Binary Odor Mixtures: Rules and Prediction
}

\author{
Yue Ma, Ke Tang, Thierry Thomas-Danguin, Yan Xu
}

\section{To cite this version:}

Yue Ma, Ke Tang, Thierry Thomas-Danguin, Yan Xu. Pleasantness of Binary Odor Mixtures: Rules and Prediction. Chemical Senses, 2020, 45 (4), pp.303-311. 10.1093/chemse/bjaa020 . hal-02880526

\section{HAL Id: hal-02880526 \\ https://hal.inrae.fr/hal-02880526}

Submitted on 25 Jun 2020

HAL is a multi-disciplinary open access archive for the deposit and dissemination of scientific research documents, whether they are published or not. The documents may come from teaching and research institutions in France or abroad, or from public or private research centers.
L'archive ouverte pluridisciplinaire HAL, est destinée au dépôt et à la diffusion de documents scientifiques de niveau recherche, publiés ou non, émanant des établissements d'enseignement et de recherche français ou étrangers, des laboratoires publics ou privés. 


\section{Pleasantness of binary odor mixtures: rules and prediction}

Yue Ma,,$+\mathrm{Ke}^{\dagger}$ Tang, $\uparrow$ Thierry Thomas-Danguin, ${ }^{*}$ Yan $\mathrm{Xu} * \dagger$

$\uparrow$ School of Biotechnology, Jiangnan University, 1800 Lihu Avenue, Wuxi, Jiangsu 214122, People's Republic of China.

$¥$ Centre des Sciences du Goût et de l’Alimentation, INRAE, CNRS, AgroSup Dijon, Université Bourgogne Franche-Comté, Dijon, France.

* To whom correspondence should be addressed:

Dr. Thierry Thomas-Danguin (Tel: +33 380 693084; Fax: +33 380 693227);

E-mail: thierry.thomas-danguin@inrae.fr

Prof. Yan Xu. (Tel: +86 510 85918197; Fax: +86 51085918201;

E-mail:yxu@jiangnan.edu.cn

(C) The Author(s) 2020. Published by Oxford University Press. All rights reserved. For permissions, please e-mail: journals.permissions@oup.com 


\section{Abstract}

2 Pleasantness is a major dimension of odor percepts. While naturally encountered odors rely on

3 mixtures of odorants, few studies have investigated the rules underlying the perceived pleasantness

4 of odor mixtures. To address this issue, a set of 222 binary mixtures based on a set of 72 odorants

5 were rated by a panel of 30 participants for odor intensity and pleasantness. In most cases, the

6 pleasantness of the binary mixtures was driven by the pleasantness and intensity of its components.

7 Nevertheless, a significant pleasantness partial addition was observed in six binary mixtures

8 consisting of two components with similar pleasantness ratings. A mathematical model, involving

9 the pleasantness of the components as well as $\tau$-values reflecting components' odor intensity, was applied to predict mixture pleasantness. Using this model, the pleasantness of mixtures including two components with contrasted intensity and pleasantness could be efficiently predicted at the

12 panel level $\left(R^{2}>0.80, R M S E<0.67\right)$. 


\section{Introduction}

The main features of odor percepts include odor intensity, odor quality, and odor pleasantness. It has been suggested that the most important one is pleasantness (hedonic dimension or valence) (Block, 2018; Rolls, Kringelbach, \& de Araujo, 2003; Wise, Olsson, \& Cain, 2000). In particular, when a wide range of odors are assessed at a similar odor intensity, the hedonic dimension is the most salient (Zarzo, 2008). The pleasantness of an odor not only affects our judgment but also causes changes in individual physiological parameters. Exposure to odors with different pleasantness levels can modify heart rate, skin conductance, and skin temperature (He, Boesveldt, de Graaf, \& de Wijk, 2014). Odor pleasantness is determined by many factors, including the molecular structure (Khan et al., 2007), odor quality (Kermen et al., 2011) and odor intensity (Doty, 1975) of the odorant; but also individual features, such as genetic (Keller, Zhuang, Chi, Vosshall, \& Matsunami, 2007) and cognitive factors, aging (Konstantinidis, Hummel, \& Larsson, 2006), culture (Ayabe-Kanamura et al., 1998; Seo et al., 2011) and physiological status (Rouby, Pouliot, \& Bensafi, 2009); and temporary environmental factors, such as visual stimuli (Hummel et al., 2017).

Most olfactory stimulation naturally occurring are mixtures of odorants (Thomas-Danguin et al., 2014). In food for instance, odor stimuli consists of 3 to 40 genuine key odorants whose composition and concentration ratios vary (Dunkel et al., 2014). In that case, perceptual interactions inducing e.g. masking or synergy add another level of complexity in food flavor understanding (Burseg \& de Jong, 2009; Escudero, Campo, Farina, Cacho, \& Ferreira, 2007; Lytra, Tempere, de Revel, \& Barbe, 2012; Ma, Tang, Xu, \& Li, 2017; Thomas-Danguin et al., 2014). Factors including the relative intensities of odorants, mixture complexity, component salience, trigeminal interactions (Walliczek-Dworschak et al., 2018), chemical structure, and possible peripheral interactions can influence odor mixture perception (Kay, Lowry, \& Jacobs, 2003). The 
analysis of binary mixture perception is the first step in understanding the perception of odor mixtures.

Attempts to explain the underlying principles of binary mixture perception have mainly investigated odor quality and intensity (Atanasova et al., 2005; Berglund \& Olsson, 1993; Ferreira, 2012a, 2012b; Laing, Panhuber, Willcox, \& Pittman, 1984; Laing \& Willcox, 1983; McNamara, Magidson, \& Linster, 2007; Miyazawa, Gallagher, Preti, \& Wise, 2009; Thomas-Danguin \& Chastrette, 2002). Nevertheless, at present, only a few studies have attempted to investigate the pleasantness of binary mixtures. In the odor mixture literature, it is widely accepted that the pleasantness of a binary mixture tends to be an intermediate value between the pleasantness values of its components (Moskowitz \& Barbe, 1977; Spence \& Guilford, 1933) and that the perceived pleasantness is highly dependent on intensity (Laing, Eddy, \& Best, 1994; Lawless, 1977). H. Lawless studied two binary mixtures, each composed of a pleasant and an unpleasant odorant at various concentration levels. He proposed a prediction model for the pleasantness of binary mixtures from the pleasantness of their constituents, weighted by their intensity (Lawless, 1977). However, until now, only a limited number of binary odor mixtures have been evaluated with regard to their pleasantness, calling into question the applicability of these rules in a wider range of odorants. Indeed, in natural products, a large range of odorants that span stimulus space have been identified. For instance, a total of 226 key food odorants were identified in 227 food samples (Dunkel et al., 2014).

The latest available research on the pleasantness of binary mixtures was conducted by Lapid et al. (Lapid, Harel, \& Sobel, 2008). By ranking the pleasantness and intensity of 5 distinct binary mixtures constructed with different ratios of the separate constituent odors, a prediction model with 
61

62

good performance was established (Lapid et al., 2008). This model proposed the possible prediction of the pleasantness of binary mixtures from the pleasantness of their separate constituents weighted by their respective perceived intensities. Interestingly, in this study, the authors observed a partial addition effect (Ferreira, 2012a; Thomas-Danguin et al., 2014) for the mixture of L-carvone and linalool at the $50-50 \%$ concentration ratio, meaning that the pleasantness of the mixture was higher than the pleasantness of the individual constituents (Lapid et al., 2008). These results underlined the key role of odor intensity in pleasantness and suggested that mixtures made of two components with similar intensities might show interesting pleasantness effects.

The aim of the present study was twofold. First, we investigated the pleasantness of a large set of binary mixtures (222 mixtures) based on a set of 72 odorants that occur in natural products, especially food products. The odorants were selected to span the stimulus space and to cover the entire range of pleasantness. The mixtures were designed to combine odorants with similar perceived intensities because we expected to observe the most interesting patterns from mixture interactions. Indeed, mixtures including a component with a high perceived intensity should have pleasantness close to that of the most intense component. Second, we adapted a mathematical model and then applied it to our dataset to predict the pleasantness of the 222 binary mixtures.

\section{Materials and methods}

\section{Subjects}

One hundred twenty-five healthy subjects between the ages of 18 and 25 were recruited from

81 Jiangnan University. Sixty-six of these subjects went through screening tests that evaluated their performance in discriminating between different odors qualities and different odor intensity levels, 
as well as their performance in logic scaling. To test their ability to evaluate odor quality and intensity, six samples comprising three different odorants at two concentration levels were provided. The subjects needed to sort these six samples into three groups based on their odor quality similarity and then rank the odor intensity of the samples within the same group. Only the subjects who answered both parts correctly, i.e., gathered the samples with the same odor quality into a group and then correctly ranked the odor intensity within the groups, were selected for the experiment. To further test subjects' scaling abilities, we provided six pictures proposed by Meilgaard et al. (Meilgaard, Carr, \& Civille, 2006). These pictures had different shadowed areas, and subjects had to evaluate the approximate area using a linear scale. The values given by subjects were compared to the correct values, and only subjects who gave substantially incorrect ratings were not selected.

All subjects provided informed consent in line with the Helsinki Declaration, and six subjects quit after the training session, leaving 60 subjects ( 41 female) to participate in the experiment. Before the main experiment, subjects participated in 2 training sessions that aimed to provide standards for intensity scale use (see below). During the main experiment, not all the subjects evaluated all the samples (hereafter called trials because each trial included 3 odorized vials); for a given sample, 30 subjects performed the evaluation. Trials were randomly assigned to the subjects, who participated in a minimum of 3 and a maximum of 15 sensory sessions, with a maximum of 3 sessions occurring per week. During a session, participants evaluated 8 to 10 trials. Subjects were paid for their participation. 
Odorants occurring in natural products were the focus. To select these odorants, we included the

226 key food odorants (KFOs) identified in Dunkel et al. (Dunkel et al., 2014) and added 548 different odorants collected in the Flavornet database (http://www.flavornet.org/). For each of the 774 odorants we obtained circa 4000 physicochemical descriptors using the Dragon ${ }^{\circledR}$ software (Talete, Milan, Italy). We finally selected 72 odorants (Supplementary Table 1) that covered the physicochemical space (Weiss et al., 2012) and were easily available from providers. Most odorants were purchased from Sigma-Aldrich China Co. (Shanghai, China) in the highest available purity, except for $p$-anisaldehyde (obtained from Fluka) and 3-mercaptohexanol (obtained from ACROS Organics). Ultimately, 198 different binary odor mixtures (Supplementary Table 2), plus 24 duplicated binary odor mixtures, made from the 72 odorants were designed for the experiments based on their odor characteristics.

\section{Equal-intensity stimuli}

All odorants were diluted with odorless solvents which were 1,2-propanediol, or mineral oil or deionized distilled water depending on odorant solubility. To avoid large differences in intensity and to keep it in a narrow range for all samples, odorants were first diluted to a point approximately equal to the odor intensity of ethyl 2-methylbutyrate at a concentration of $3.9 \mathrm{~g} / \mathrm{L}$, as estimated by experienced lab members. Then, we prepared a set of solutions of odorants varying around the obtained concentration. These solutions were presented to 6 subjects who did not participate in the main experiment and who were instructed to provide a number between 0 and 7 reflecting the solution odor intensity. For each odorant, the final concentration (Supplementary Table 1) was set to match similar intensity following the procedure described in Weiss et al. (Weiss et al., 2012). 
To prevent the formation of novel chemicals in the mixtures, odorants were not mixed in the liquid phase. For the unmixed odor samples, $200 \mu \mathrm{L}$ of diluted stimulus was poured onto a $0.1 \mathrm{~g}$ cotton ball and placed in a $20 \mathrm{~mL}$ brown glass bottle. For the binary mixtures, $200 \mu \mathrm{L}$ of each stimulus was poured onto separate sides of the $0.1 \mathrm{~g}$ cotton ball, such that the two odorants' vapors alone mixed in the glass bottle headspace. All of the stimuli were fully absorbed by the cotton ball. All samples were prepared one day before the sensory session and stored at room temperature $\left(24^{\circ} \mathrm{C}\right)$.

\section{General procedures}

The data presented in this study include 33,300 psychophysical single evaluations collected from fifteen sessions across three months (Figure 1). Before the formal experiment, we began with two training sessions. The first session determined the standard odor references to be used in the experiment. Ethyl 2-methylbutyrate and linalool were selected as reference odorants because the majority of panelists did not object to sniff it frequently, and because their corresponding odors (fruity-green-apple and floral-citrus-lavender respectively) were rather familiar to the participants, references, we gave participants ethyl 2-methylbutyrate $(1.8 \mathrm{~g} / \mathrm{L})$ and linalool $(10.7 \mathrm{~g} / \mathrm{L})$ and asked them to rate the intensity of these two samples. We asked them to evaluate ethyl 2-methylbutyrate first, and then, they need to evaluate the intensity of linalool by comparing the intensity of linalool with the intensity of ethyl 2-methylbutyrate. If the intensity of linalool smelled twice as strong as ethyl 2-methylbutyrate, its intensity was marked twice the distance from zero as the position of 
ratings across all subjects. The intensities of standard I (ethyl 2-methylbutyrate) and standard II

149 (linalool) were finally anchored as 3.0 and 7.0, respectively. The second session introduced the odor 150 evaluation procedures. During this session, the two standards were provided to the subjects, and 151 they were told that they had to rate the perceived intensity of the samples presented during the 152 formal sessions using the anchor intensities of the two standards.

153

154

155

156

157

158

159

160

In the formal sessions, a total of 222 trials, among which 24 were duplicated trials, were

evaluated (Figure 1). Each session in the formal experiment comprised 14 to 15 trials, and each trial included three stimuli: two stimuli were single odorants, and the third stimulus was a binary mixture of these odorants. In each trial, all the unmixed odor samples were coded by three random digits, and the binary mixture sample was coded by its trial number. The order of the presentation of the two unmixed odors was counterbalanced for each trial. Subjects were given a rest of 45 seconds between each stimulus. Each trial was presented to subjects in a random order, and one trial was evaluated by a maximum of 30 subjects.

\section{Each session included three parts. The first part consisted of a hedonic evaluation, and the last} two parts consisted of intensity evaluations. During the hedonic evaluation, subjects had to mark off distance on a visual analog scale 100-mm in length (Figure 1). For the intensity evaluations, in order to get the panelists used to the scale in a similar way across the range of intensity, an adjusted explicit anchoring scale was used by marking the position of standard I and standard II. The two standards were determined in the training session, and were presented in the first two sessions to help the subjects rate odor intensity. This kind of anchoring scale with reference standards has a long history in texture analysis, which might generate more reliable sensory data by reducing the variability among panelists both in evaluation procedures and individual judgments, as well as 
within panelists in replicated assessments (Muñoz, 1986). Subjects had to mark off distance on the

171 visual analog scale according to the perceptual anchors. If the stimulus smelled twice as strong as

172

173

174

175

176

the standard, its intensity was marked twice the distance from zero as the standard position. If the test stimulus smelled half as strong as the standard, its intensity was marked half the distance, and so on (Figure 1). In the second part of the intensity evaluation session, subjects had to evaluate the intensity of the unmixed components perceived in the binary mixture and whether they could perceive a new odor in the mixture (data not shown).

\section{Data processing}

Psychophysical data obtained from the scales were transformed into numerical values. All the scores within the range of the scale were translated to a value between 0.0 and 10.0. Statistical analyses were performed with R software (version 3.5.3).

\section{Panel performance was checked through principal component analysis (PCA). Nonparametric} Mixed Effects ANOVA was applied to test the repeatability of 24 duplicated trials for each intensity variable using the lmer and glmer functions from the lme4 package (Bates, Mächler, Bolker, \& Walker, 2014). The subject effect and trial effect were set as random factors. Differences between trials were analyzed using an unpaired two-sample Wilcoxon test using the wilcox.test function from the ddply package (Wickham, 2011). The averages across subjects of the intensity or pleasantness of each odorant were compared to the mean values across odorants using unpaired Wilcoxon test from the ggpubr package (Kassambara, 2019). The difference between odor intensity (resp. pleasantness) of a binary mixture and its two components in each trial was analyzed using a paired Wilcoxon test (wilcox.test function). Bonferroni correction was applied to account for multiple testing when necessary. 

intensity of odor A or odor B to the sum of their intensities (Patte \& Laffort, 1979), was applied to predict the binary mixture pleasantness.

$$
\begin{gathered}
P_{A B}=\tau_{A} P_{A}+\tau_{B} P_{B} \\
\tau_{A}=\frac{I_{A}}{I_{A}+I_{B}} \text { or } \tau_{B}=\frac{I_{B}}{I_{A}+I_{B}}
\end{gathered}
$$

195 196

This model was equivalent to the intensity weights model proposed by Lapid et al. (Lapid et al., 2008). The performance of this prediction model was tested by computing the prediction error Root Mean Squared Error $(R M S E)$ and the R-square $\left(R^{2}\right)$ between experimental and predicted values. This model was applied to predict the mixture odor pleasantness at panel level as well as individual level. In the panel approach, a single pleasantness value was predicted for a given mixture, while in the individual approach, a pleasantness value was predicted for each subject for a given mixture. In addition, for the panel approach, predicted pleasantness was calculated using either the average pleasantness across trials and mean $\tau$-value of the 2 components (mean condition, Equation 1) or using the average pleasantness across subjects and $\tau$-values of each trial (trial condition, Equation 2).

$P_{A B \_ \text {mean }}=\tau_{A \_ \text {mean }} P_{A_{-} \text {mean }}+\tau_{B_{-} \text {mean }} P_{B_{-} \text {mean }}$ Equation 1

$P_{A B_{-} \text {trial }}=\tau_{A_{-} \text {trial }} P_{A_{-} \text {trial }}+\tau_{B_{-} \text {trial }} P_{B_{-} \text {trial }}$ Equation 2 condition of the panel approach, a single value of pleasantness and a single value of $\tau$ were calculated and used to predict the pleasantness of all the mixtures including this odorant. In contrast, in the trial condition of the panel approach, one average value of pleasantness and one $\tau$ 
value across subjects were calculated for each trial to predict one value of mixture pleasantness per 212 trial.

In the individual approach, we also considered the two conditions mean and trial. Thus, predicted 214 pleasantness was calculated for each subject using either the average pleasantness across trials and mean $\tau$-value of the 2 components (mean condition, Equation 3) or using the pleasantness value and $\tau$-value from a given subject on each trial (trial condition, Equation 4).

For example, for ethyl 2-methylbutyrate in the mean condition of the individual approach, an individual value of pleasantness and $\tau$ was calculated across trials and was used to predict the individual mean pleasantness of mixtures including this odorant, whereas in the trial condition of the individual approach, one value of pleasantness and $\tau$ was calculated per trial for each subject. cor.test function, the prediction performances of the three models were compared based on the Pearson's product moment correlation coefficient between predicted and experimental pleasantness and the 95 percent confidence interval on this correlation coefficient. The formula of each model

In addition to the Tau-based model (intensity weights model), the squared model and the sin model (Lapid et al., 2008) were applied to predict the binary mixture pleasantness. Using the and the correlation results were provided in Supplement Table 3. The results showed that there was no significant difference between the three models since there is an overlap of the 95 percent 
confidence interval within each prediction approach/condition. Hereafter, only the simplest Taubased model was considered.

\section{Results and Discussion}

\section{Panel performance and repeatability}

The subjects' overall performance and coherence were checked using PCA on the raw data. The PCA map of individuals for the first 2 dimensions, explaining $16.7 \%$ of the total variance, is reported in Supplementary Figure 1. We checked the individual results from the subjects outside of the central cloud for the different variables more in depth, and we did not identify any systematic outliers. Therefore, all the data were kept for further analyses.

Nonparametric Mixed Effects ANOVA was applied to test repeatability using the 24 duplicated trials for each attribute. Variables included the intensity of odor $A\left(I_{A}\right)$ or odor $B\left(I_{B}\right)$, the pleasantness of odor $\mathrm{A}\left(\mathrm{P}_{\mathrm{A}}\right)$ or odor $\mathrm{B}\left(\mathrm{P}_{\mathrm{B}}\right)$, and the pleasantness of the binary mixture $\left(\mathrm{P}_{\mathrm{AB}}\right)$. The results indicated no significant repetition effects $(p>0.05)$, except for the pleasantness of odor A $\left(\mathrm{P}_{\mathrm{A}}, p<0.001\right)$. By checking the repeatability of attributes $\mathrm{P}_{\mathrm{A}}$ for each repeated trial, only the means of Trial 36 (ethyl 2-methylbutyrate, Supplementary Table 2) was found to be significantly different between the replicates (Wilcoxon-test with Bonferroni correction). Ethyl 2-methylbutyrate was used 29 times in the whole experiment (Supplementary Table 1, and Supplementary Figure 2); thus, the pleasantness rating might have evolved as a result of increasing familiarity with the odor of this compound. Although the pleasantness of ethyl 2-methylbutyrate might have been overrated at the end of the pleasantness evaluation, the statistical assessment showed that the panel could rate odor 
253

intensity and pleasantness consistently and consensually in most cases and that the psychophysical data were statistically reliable.

\section{Binary odor pleasantness perception}

The mean intensity and pleasantness of each odorant were calculated across subjects in all trials

(Figure 2). Uncorrected unpaired Wilcoxon test was used to test the difference between the intensity of each odorant and the mean intensity value across odorants. Although we tried to provide stimuli that had similar intensities (preliminary test with external panel of six subjects), the results showed that there were 19 out of 72 odorants whose odor intensity was significantly different from the mean value $(p<0.001)$. Among these odorants, ethyl octanoate, $o$-aminoacetophenone, ethyl valerate, $p$ cresol, $\gamma$-undecalactone, butanal, pentanal, phenylethylthiol and benzaldehyde had intensities that were significantly higher than the mean intensity $(p<0.001)$, with intensities ranging from 6.53 to 7.71, while the intensities of ethyl laurate, undecanaldehyde, 2-pentanone, vanillin, $\gamma$-butyrolactone, eugenol, ethyl 3-(methylsulfanyl)propanoate, nerol oxide, carveol, geraniol and isoeugenol were significantly lower than the mean intensity $(p<0.001)$, with intensities ranging from 3.68 to 5.15

(Supplementary Figure 3). Uncorrected unpaired Wilcoxon test was also used to test the difference between the pleasantness value of each odorant and the mean value, and there were 19 odorants whose odor pleasantness was significantly different from the mean value $(p<0.001)$

\section{(Supplementary Figure 3).}

Uncorrected paired Wilcoxon test was used to evaluate the difference in intensity and pleasantness between the two components of each of the 198 different binary odor samples (trials).

As a result, four groups of trials were considered. First, group E, comprising 50 trials, showed no significant difference in either intensity or pleasantness $(p<0.05)$; group I, which included 52 
trials, showed a significant difference in intensity only $(p<0.05)$; group $\mathrm{P}$, comprising 39 trials, showed a significant difference in pleasantness only $(p<0.05)$; and finally, group IP, comprising the remaining 57 trials, showed a significant difference in both intensity and pleasantness $(p<0.05)$ was obtained (Supplementary Table 2).

The results of pleasantness rating of the 198 binary odor mixtures showed that, in most cases, mixture pleasantness was in-between pleasantness of the unmixed odorants (Figure 3), and that mixture pleasantness scores varied according to pleasantness and intensity scores of the unmixed odorants. If the binary mixture consisted of two components with contrasted pleasantness and intensity (group IP), the pleasantness of the binary mixture was generally closer to that of the stronger odor component. For example, in the trial with ethyl valerate and $p$-cymene (Trial 2), the pleasantness (5.39) and intensity (7.19) of ethyl valerate were higher than the pleasantness (4.06) and intensity (5.21) of $p$-cymene, and the pleasantness of the binary odor (5.27) was closer to that of ethyl valerate. In the trial with 1-heptanol and phenylethylthiol (Trial 12), the pleasantness of 1heptanol (4.58) was higher than that of phenylethylthiol (2.45), but its intensity (4.58) was weaker than that of phenylethylthiol (7.43). The pleasantness of the binary odor (2.34) was almost the same as that of phenylethylthiol. However, this pattern did not apply for all trials, such as that with $\gamma-$ heptalactone and diethyl acetal (Trial 150), vanillin and diethyl acetal (Trial 153) or ethyl 3methylbutanoate and ethyl isobutyrate (Trial 160). In the trial with vanillin and diethyl acetal, there were significant differences in both the intensity and pleasantness of these two odorants; the intensity of vanillin (4.03) was weaker than that of diethyl acetal (5.40), but the pleasantness of the binary odor (6.52) was closer to that of vanillin (6.48) than diethyl acetal (4.74). This phenomenon might have resulted from perceptual interactions at the intensity level. For instance, a masking effect caused by vanillin could reduce the intensity of the odor of diethyl acetal in the mixture due 
to the perceptual dominance of the vanillin odor quality (Atanasova et al., 2005), and therefore, the pleasantness of the mixture would be closer to that of vanillin alone.

If the binary odor mixture included two odorants with contrasted pleasantness but almost the same intensity (group P), the pleasantness of the binary mixture was generally near the mean pleasantness or was closer to the lower pleasantness value of the two odors. This phenomenon was observed in most trials, except for those with 2-octanone and ethyl butyrate (Trial 121), hexyl hexanoate and geranyl acetate (Trial 171), geraniol and ethyl butyrate (Trial 183), and ethyl butyrate and hexanal (Trial 198). In these four trials, the pleasantness of the binary mixture was close to the highest pleasantness value of the two odors. This specific case might result from perceptual interactions such as masking, synergy (Ferreira, 2012a) or perceptual dominance (Atanasova et al., 2005), which may affect odor intensity and/or odor quality of the odor mixture and consequently its pleasantness.

Overall, for mixtures including a pleasant and a less pleasant component, we observed, in most of the cases, that the stronger constituent was more influential on the mixture's pleasantness than the weaker one. This rule is in accordance with previous observations (Laing et al., 1994; Lapid et al., 2008; Lawless, 1977; Moskowitz \& Barbe, 1977; Spence \& Guilford, 1933). Moreover, the weight of this influence was stronger for unpleasant components, as previously reported (Lawless, 1977).

However, the special cases observed in group IP and group P also indicated that the pleasantness of binary mixtures is driven by the intensity of each component perceived within the mixture rather than by the intensity perceived out of the mixture. Indeed, mixing at least two odors can lead to several quantitative and qualitative effects on the mixture odor (Berglund \& Olsson, 1993) and/or quality effects (e.g., perceptual dominance (Atanasova et al., 2005)) that further influence the odor 
pleasantness of the mixture. These perceptual interactions can arise from several biochemical or neurobiological interactions during all stages of olfactory information processing within the olfactory system, from the periphery to the brain (Thomas-Danguin et al., 2014). As odor pleasantness is believed to be partially innate, but also strongly shaped by experience and learning (Prescott, Kim, \& Kim, 2008), an odorant with higher recognition or carrying nutritious or poisonous information might capture more attention in a binary mixture (White, Thomass-Danguin, Olofsson, Zucco, \& Prescott, 2020) and these factors might play an important role in the pleasantness judgement of the binary mixture. Indeed, the attentional capture effect has been highlighted in brain imaging studies using a binary odor mixture including a pleasant and an unpleasant component (Grabenhorst, Rolls, \& Margot, 2011; Grabenhorst, Rolls, Margot, da Silva, \& Velazco, 2007).

If the binary mixture consisting of two components with similar pleasantness (group I and group E), the pleasantness of the binary mixture was, in most cases, the same as that of the components, but we also observed several cases indicating partial addition. A partial addition effect means that the pleasantness of the mixture is higher than the pleasantness of each component individually or that the pleasantness of the mixture is lower than that of each component individually. In the latter case, one can consider this effect as partial addition for unpleasantness. In our dataset, we observed that there were 52 trials (26\%) showing partial additive pleasantness, meaning that the pleasantness of the binary mixtures was higher than either of its components, and 28 trials (14\%) in which partial additive unpleasantness occurred (e.g., the pleasantness of the mixture was lower than that of either of its components). The statistical significance of the pleasantness partial addition effect for each trial was tested by uncorrected paired samples Wilcoxon tests. If there were significant differences between the pleasantness of each component $\left(\mathrm{P}_{\mathrm{A}}\right.$ or $\left.\mathrm{P}_{\mathrm{B}}\right)$ and the pleasantness of the mixture $\left(\mathrm{P}_{\mathrm{AB}}\right)$, 
and if the pleasantness of the mixture was lower than the sum of pleasantness score of each component $\left(\mathrm{P}_{\mathrm{A}}+\mathrm{P}_{\mathrm{B}}\right)$, we considered that the pleasantness partial addition effect was significant. There were 6 trials with significant partial additive pleasantness: methyl octanoate and ethyl 2methylbutyrate (Trial 61, $p<0.05$ ), ethyl octanoate and benzyl acetate (Trial 83, $p<0.01$ ), isoeugenol and $\gamma$-decalactone (Trial 188, $p<0.05$ ), 1,8-cineole and ethyl valerate (Trial 196, $p<$ 0.05), linalool and 2-octanone (Trial 206, $p<0.01$ ), and eugenol and 2-octanone (Trial 217, $p<$ 0.05) (Supplementary Table 2). Here, significant partial additive pleasantness was only observed in five binary mixtures consisting of two components with similar pleasantness and intensity and in one binary mixture consisting of two components with similar pleasantness but different intensity. Significant partial additive pleasantness was observed in a mixture of $L$-carvone and linalool at a $50-50 \%$ concentration ratio in Lapid's study (Lapid et al., 2008). It is interesting to consider that at the $50-50 \%$ concentration ratio, the intensity and pleasantness of $L$-carvone and linalool were also similar in the abovementioned study. Therefore, it is reasonable to propose that pleasantness partial addition might tend to occur in mixtures with two components of similar pleasantness and similar intensity. One speculation that can explain partial additive pleasantness would consider an additive effect in the intensity of the mixture (Lapid et al., 2008), but the underlying principles of the effects need to be investigated more in depth through a systematic study of more binary mixtures of that kind.

There were 28 trials in which partial additive unpleasantness was observed, but none of them were found to reach a statistically significant level. A study (Laing et al., 1994) investigated the interactions between four sewage-related unpleasant odorants: hydrogen sulphide, isovaleric acid, butanethiol, and skatole. In this research, the pleasantness of a mixture was lower than the pleasantness of the individual (unmixed) components in most instances (Laing et al., 1994). Thus, 
based on this result, we assumed that the unpleasantness of a mixture might be stronger than that of the individual constituents if the binary mixture consists of two extremely unpleasant odorants. In our dataset, several binary mixtures included two components with extremely unpleasant odors (e.g., Trial 34, 40, 66, and 154). Nevertheless, the unpleasantness of these binary mixtures was not stronger than that of the individual constituents. Another example of partial additive unpleasantness was observed in the mixture of butanoic acid and phenylethyl alcohol, even though the effect was not significant (Lapid et al., 2008). The author speculated that partial additive unpleasantness might occur in cases in which at least one of the components shows a steep decline in pleasantness as a function of its intensity and an increase in the intensity of the mixture above the intensity of its constituents (Lapid et al., 2008).

\section{Pleasantness prediction}

A model based on the $\tau$-value proposed by Patte and Laffort (Patte \& Laffort, 1979) reflecting the relative proportion of the perceived intensity of odor A or odor B in a mixture was applied to predict the pleasantness of binary mixtures. This model was equivalent to the intensity weights model one used by Lapid et al. (Lapid et al., 2008). This model was applied to predict mixture odor pleasantness not only at the panel level but also at the individual level. In addition, for panel and individual approaches, predicted pleasantness was calculated as a mean condition and as a trial condition to check whether the differences in pleasantness and intensity that may arise for a given pair of odors (i.e., within a trial) have an impact on the mixture pleasantness rating or, in contrast, if pleasantness and intensity might be considered as properties of the compounds (i.e., mean) regardless of the odor pair. The model performance was evaluated by computing the prediction error $R M S E$ and the $R^{2}$. RMSE represents the average difference between the perceptual 
pleasantness in trials and the predicted pleasantness by the model. The $R^{2}$ represents the correlation between the perceptual pleasantness and the predicted pleasantness. The lower the RMSE and the higher the $R^{2}$ are, the better the model.

The performance of the model for the panel approach obtained for the mean condition and the trial condition are shown in Figure 4a. In the panel approach, for all the trials in the trial condition, the $R^{2}$ was 0.857 , the prediction error $R M S E$ was 0.428 , whereas in the mean condition, the $R^{2}$ was 0.732 , and the prediction error RMSE was 0.584 , meaning that the prediction in the trial condition was better than that in the mean condition. Then, the prediction model was used to predict the pleasantness of the four trial groups we defined above (group E, group I, group IP, group P). The results (Table 1) showed that in the trial condition, the prediction model performed quite well regardless of the group $\left(R^{2}>0.80\right)$. However, in the mean condition, only group IP obtained a high $R^{2}$ value. This result showed that the model performance in predicting group IP was high, meaning that the model based on the $\tau$-value predicting the pleasantness of a binary mixture consisting of two components with contrasted intensity and pleasantness performed quite well regardless of the odor pair. For group I, in which components had contrasted intensity, the $R^{2}$ value was low, but the prediction error RMSE was also low; in this case, it is likely that only a few instances of poor prediction might have been observed. For every group, especially for groups I, P and E, the model performance in the mean condition was worse than that in the trial condition. This result suggests that a context effect existed for specific combinations. The context effect, which implies that the perception of one odorant is influenced by the other odorant in the pair, might be an influential factor for pleasantness, especially for couples of odorants with similar odor pleasantness or intensity. In the future, at the panel level, an improved prediction model for the pleasantness of binary mixtures of two components with similar intensity or pleasantness must take into account the 
context effect, for instance, considering specific chemical features or specific odor quality features of the mixed odorants, to be able to account for additive effects.

This model was then used to determine whether the individual pleasantness of a given binary odor can be predicted (Figure 4b). Compared with the prediction in the panel approach, the predictions in the individual approach were relatively poor regardless of the condition (trial or the mean) and regardless of the trial group (Table 3). The significant variance and poor predictive performance of the model specified that predictions at an individual level are still a major challenge.

This difficulty might be due to the high interindividual variability in odor pleasantness (Lindqvist, Hoglund, \& Berglund, 2012), supported by individual genetic and cognitive differences. Indeed, previous research has shown that genetic variation across the human olfactory receptor repertoire alters odor perception in the intensity and pleasantness of a given odor (Keller et al., 2007; Trimmer et al., 2019), and stimulus intensity, repeated exposure, sex and hormonal status, aging, emotional status, and cultural background can all influence individual pleasantness ratings (Rouby et al., 2009).

\section{Conclusions}

On the basis of a sample set of 198 different binary odor mixtures, we showed that when two odorants are mixed, the pleasantness of the binary mixture follows different rules: 1) If two odorants with significantly different intensity were mixed, in most cases, the pleasantness of the binary mixture was closer to that of the strongest odor component. 2) If two odorants with similar intensity but contrasted pleasantness were mixed, the pleasantness of binary mixture was generally near the mean pleasantness or was closer to that of the odor with the lower pleasantness value. 3) 
433 Partial additive pleasantness tended to occur in mixtures of two components with similar

434 pleasantness and intensity ratings. We highlighted that a model based on the $\tau$-value predicting the 435 pleasantness of a binary mixture consisting of two components with contrasted or similar intensity 436 and pleasantness performed quite well regardless of the odor pair, whereas prediction at the 437 individual level was still a major challenge. In future studies, it would be interesting to use this 438 model to predict the pleasantness of larger mixtures, while considering them as a series of binary 439 mixtures. 
443 This work was supported by the National Key R\&D Program (2016YFD0400504), National 444 First-class Discipline Program of Light Industry Technology and Engineering (LITE 2018-12), 445 China Scholarship Council (201806790033), and Postgraduate Research \& Practice Innovation 446 Program of Jiangsu Provence (KYCX18_1788).

\section{Acknowledgments}

448 The authors are grateful to all the subjects for participating in the psychophysical experiment.

449

450 


\section{References}

Atanasova, B., Thomas-Danguin, T., Chabanet, C., Langlois, D., Nicklaus, S., \& Etievant, P. (2005). Perceptual interactions in odour mixtures: odour quality in binary mixtures of woody and fruity wine odorants. Chem. Senses, 30(3), 209-217. doi:10.1093/chemse/bji016

Ayabe-Kanamura, S., Schicker, I., Laska, M., Hudson, R., Distel, H., Kobayakawa, T., \& Saito, S. (1998). Differences in perception of everyday odors: a Japanese-German cross-cultural study. Chem. Senses, 23(1), 31-38. doi:10.1093/chemse/23.1.31

Bates, D., Mächler, M., Bolker, B., \& Walker, S. (2014). Fitting linear mixed-effects models using 1me4. arXiv preprint arXiv, 1406(5823).

Berglund, B., \& Olsson, M. J. (1993). Odor-intensity interaction in binary and ternary mixtures. Percept. Psychophys., 53(5), 475-482. doi:10.3758/bf03205195

Block, E. (2018). Molecular basis of mammalian odor discrimination: a status report. J. Agric. Food Chem., 66(51), 13346-13366. doi:10.1021/acs.jafc.8b04471

Burseg, K., \& de Jong, C. (2009). Application of the Olfactoscan method to study the ability of saturated aldehydes in masking the odor of methional. J. Agric. Food Chem., 57(19), 90869090. doi:10.1021/jf9016866

Doty, R. L. (1975). An examination of relationships between the pleasantness, intensity, and concentration of 10 odorous stimuli. Percept. Psychophys., 17(5), 492-496.

Dunkel, A., Steinhaus, M., Kotthoff, M., Nowak, B., Krautwurst, D., Schieberle, P., \& Hofmann, T. (2014). Nature's chemical signatures in human olfaction: a foodborne perspective for future biotechnology. Angew. Chem.-Int. Edit., 53(28), 7124-7143. doi:10.1002/anie.201309508

Escudero, A., Campo, E., Farina, L., Cacho, J., \& Ferreira, V. (2007). Analytical characterization of the aroma of five premium red wines. Insights into the role of odor families and the concept 
of fruitiness of wines. Journal of agricultural and food chemistry, 55(11), 4501-4510.

475

476

477

478

479

480

481

482

483

484

485

486

487

488

489

490

491

492

493

494

495

496 doi:10.1021/jf0636418

Ferreira, V. (2012a). Revisiting psychophysical work on the quantitative and qualitative odour properties of simple odour mixtures: a flavour chemistry view. Part 1: intensity and detectability. A review. Flavour Frag. J., 27(2), 124-140. doi:10.1002/ffj.2090

Ferreira, V. (2012b). Revisiting psychophysical work on the quantitative and qualitative odour properties of simple odour mixtures: a flavour chemistry view. Part 2: qualitative aspects. A review. Flavour Frag. J., 27(3), 201-215. doi:10.1002/ffj.2091

Grabenhorst, F., Rolls, E. T., \& Margot, C. (2011). A hedonically complex odor mixture produces an attentional capture effect in the brain. Neuroimage, 55(2), 832-843. doi:10.1016/j.neuroimage.2010.12.023

Grabenhorst, F., Rolls, E. T., Margot, C., da Silva, M., \& Velazco, M. I. (2007). How pleasant and unpleasant stimuli combine in different brain regions: odor mixtures. J. Neurosci., 27(49), 13532-13540. doi:10.1523/jneurosci.3337-07.2007

He, W., Boesveldt, S., de Graaf, C., \& de Wijk, R. A. (2014). Dynamics of autonomic nervous system responses and facial expressions to odors. Front. Psychol., 5, 110. doi:10.3389/fpsyg.2014.00110

Hummel, T., Fark, T., Baum, D., Warr, J., Hummel, C. B., \& Schriever, V. A. (2017). The rewarding effect of pictures with positive emotional connotation upon perception and processing of pleasant odors-an fMRI study. Front. Neuroanat., 11, 19. doi:10.3389/fnana.2017.00019

Kassambara, A. (2019). ggpubr:“ggplot2” based publication ready plots (Version R Package Version $0.2 ; 2018$.$) .$

Kay, L. M., Lowry, C. A., \& Jacobs, H. A. (2003). Receptor contributions to configural and 
elemental odor mixture perception. Behav. Neurosci., 117(5), 1108-1114. doi:10.1037/07357044.117.5.1108

Keller, A., Zhuang, H. Y., Chi, Q. Y., Vosshall, L. B., \& Matsunami, H. (2007). Genetic variation in a human odorant receptor alters odour perception. Nature, 449(7161), 468-472. doi:10.1038/nature06162

Kermen, F., Chakirian, A., Sezille, C., Joussain, P., Le Goff, G., Ziessel, A., . . Bensafi, M. (2011). Molecular complexity determines the number of olfactory notes and the pleasantness of smells. Sci. Rep., 1, 206. doi:10.1038/srep00206

Khan, R. M., Luk, C. H., Flinker, A., Aggarwal, A., Lapid, H., Haddad, R., \& Sobel, N. (2007). Predicting odor pleasantness from odorant structure: pleasantness as a reflection of the physical world. J. Neurosci., 27(37), 10015-10023. doi:10.1523/JNEUROSCI.1158-07.2007

Konstantinidis, I., Hummel, T., \& Larsson, M. (2006). Identification of unpleasant odors is independent of age. Arch. Clin. Neuropsychol., 21(7), 615-621. doi:10.1016/j.acn.2006.05.006

Laing, D. G., Eddy, A., \& Best, D. J. (1994). Perceptual characteristics of binary, trinary, and quaternary odor mixtures consisting of unpleasant constituents. Physiol. Behav., 56(1), 8193.

Laing, D. G., Panhuber, H., Willcox, M. E., \& Pittman, E. A. (1984). Quality and intensity of binary odor mixtures. Physiol. Behav., 33(2), 309-319. doi:10.1016/0031-9384(84)90118-5

Laing, D. G., \& Willcox, M. E. (1983). Perception of components in binary odor mixtures. Chem. Senses, 7(3-4), 249-264. doi:10.1093/chemse/7.3-4.249

Lapid, H., Harel, D., \& Sobel, N. (2008). Prediction models for the pleasantness of binary mixtures in olfaction. Chem. Senses, 33(7), 599-609. doi:10.1093/chemse/bjn026 
Lawless, H. T. (1977). The pleasantness of mixtures in taste and olfaction. Sensory Processes, 1, 227-237.

Lindqvist, A., Hoglund, A., \& Berglund, B. (2012). The role of odour quality in the perception of binary and higher-order mixtures. Perception, 41(11), 1373-1391. doi:10.1068/p7267

Lytra, G., Tempere, S., de Revel, G., \& Barbe, J. C. (2012). Impact of perceptive interactions on red wine fruity aroma. J. Agric. Food Chem., 60(50), 12260-12269. doi:10.1021/jf302918q

Ma, Y., Tang, K., Xu, Y., \& Li, J.-m. (2017). Characterization of the key aroma compounds in Chinese Vidal icewine by gas chromatography-olfactometry, quantitative measurements, aroma recombination, and omission tests. Journal of agricultural and food chemistry, 65(2), 394-401. doi:10.1021/acs.jafc.6b04509

McNamara, A. M., Magidson, P. D., \& Linster, C. (2007). Binary mixture perception is affected by concentration of odor components. Behav. Neurosci., 121(5), 1132-1136. doi:10.1037/07357044.121.5.1132

Meilgaard, M. C., Carr, B. T., \& Civille, G. V. (2006). Selection and training of panel members Sensory evaluation techniques (pp. 174-176). New York: CRC Press.

Miyazawa, T., Gallagher, M., Preti, G., \& Wise, P. M. (2009). Odor detection of mixtures of homologous carboxylic acids and coffee aroma compounds by humans. J. Agric. Food Chem., 57(21), 9895-9901. doi:10.1021/jf901453r

Moskowitz, H. R., \& Barbe, C. D. (1977). Profiling of odor components and their mixtures. Sensory Processes, 1(3), 212-226.

Muñoz, A. M. (1986). Development and application of texture reference scales. J. Sens. Stud., 1(1), 55-83.

Patte, F., \& Laffort, P. (1979). An alternative model of olfactory quantitative interaction in binary 
544 Prescott, J., Kim, H., \& Kim, K.-O. (2008). Cognitive Mediation of Hedonic Changes to Odors Following Exposure. Chemosens. Percept., 1(1), 2-8. doi:10.1007/s12078-007-9004-y

Rolls, E. T., Kringelbach, M. L., \& de Araujo, I. E. T. (2003). Different representations of pleasant and unpleasant odours in the human brain. Eur. J. Neurosci., 18(3), 695-703. doi:10.1046/j.1460-9568.2003.02779.x

Rouby, C., Pouliot, S., \& Bensafi, M. (2009). Odor hedonics and their modulators. Food. Qual. Prefer., 20(8), 545-549. doi:10.1016/j.foodqual.2009.05.004

Seo, H. S., Guarneros, M., Hudson, R., Distel, H., Min, B. C., Kang, J. K., . . . Hummel, T. (2011). Attitudes toward olfaction: a cross-regional study. Chem. Senses, 36(2), 177-187. doi:10.1093/chemse/bjq112

Spence, W., \& Guilford, J. P. (1933). The affective value of combinations of odors. Am. J. Psychol., $45(3), 495-501$.

Thomas-Danguin, T., \& Chastrette, M. (2002). Odour intensity of binary mixtures of odorous compounds. C. R. Biol., 325(7), 767-772. doi:10.1016/s1631-0691(02)01485-3

558 Thomas-Danguin, T., Sinding, C., Romagny, S., El Mountassir, F., Atanasova, B., Le Berre, E., . . . 559 Coureaud, G. (2014). The perception of odor objects in everyday life: a review on the processing of odor mixtures. Front. Psychol., 5, 504. doi:10.3389/fpsyg.2014.00504

561 Trimmer, C., Keller, A., Murphy, N. R., Snyder, L. L., Willer, J. R., Nagai, M. H., . . Mainland, J. 562 D. (2019). Genetic variation across the human olfactory receptor repertoire alters odor perception. Proc. Natl. Acad. Sci. U. S. A., 116(19), 9475-9480. doi:10.1073/pnas.1804106115 

(2018). The presentation of olfactory-trigeminal mixed stimuli increases the response to subsequent olfactory stimuli. J. Agric. Food Chem., 66(10), 2312-2318. doi:10.1021/acs.jafc.6b04342

Weiss, T., Snitz, K., Yablonka, A., Khan, R. M., Gafsou, D., Schneidman, E., \& Sobel, N. (2012). Perceptual convergence of multi-component mixtures in olfaction implies an olfactory white. Proc. Natl. Acad. Sci. U. S. A., 109(49), 19959-19964. doi:10.1073/pnas.1208110109

White, T. L., Thomas-Danguin, T., Olofsson, J. K., Zucco, G. M., \& Prescott, J. (2020). Thought for food: Cognitive influences on chemosensory perceptions and preferences. Food. Qual. Prefer., 79, 103776. doi:10.1016/j.foodqual.2019.103776

Wickham, H. (2011). The split-apply-combine strategy for data analysis. J. Stat. Softw., 40(1), 1-29.

Wise, P. M., Olsson, M. J., \& Cain, W. S. (2000). Quantification of odor quality. Chem. Senses, $25(4), 429-443$.

Zarzo, M. (2008). Psychologic dimensions in the perception of everyday odors: pleasantness and edibility. J. Sens. Stud., 23(3), 354-376. doi:10.1111/j.1745-459X.2008.00160.x 
582 Figure 1 Schematic diagram of psychophysical experiment data collection

583 Figure 2 Intensity and pleasantness of 72 odorants calculated across all the subjects in all the trials

584 Figure 3 Pleasantness of 198 different binary odor mixtures based on 72 different odorants. The top

585 left triangle represents the value of the standard deviation; the bottom right triangle represents the

586 value of the mean pleasantness. The pleasantness values of unmixed odorants are reported on the

587 axes and correspond to the mean value shown in Figure 2. The data from the 24 duplicated trials

588 were not included.

$589 \quad$ Figure $4 \tau$-value-based model prediction of binary mixtures in the (a) panel approach and (b)

590 individual approach for the mean condition and the trial condition. 
591 Table 1 Prediction Model Performances for Four Trial Groups Calculated with Different

592 Approaches

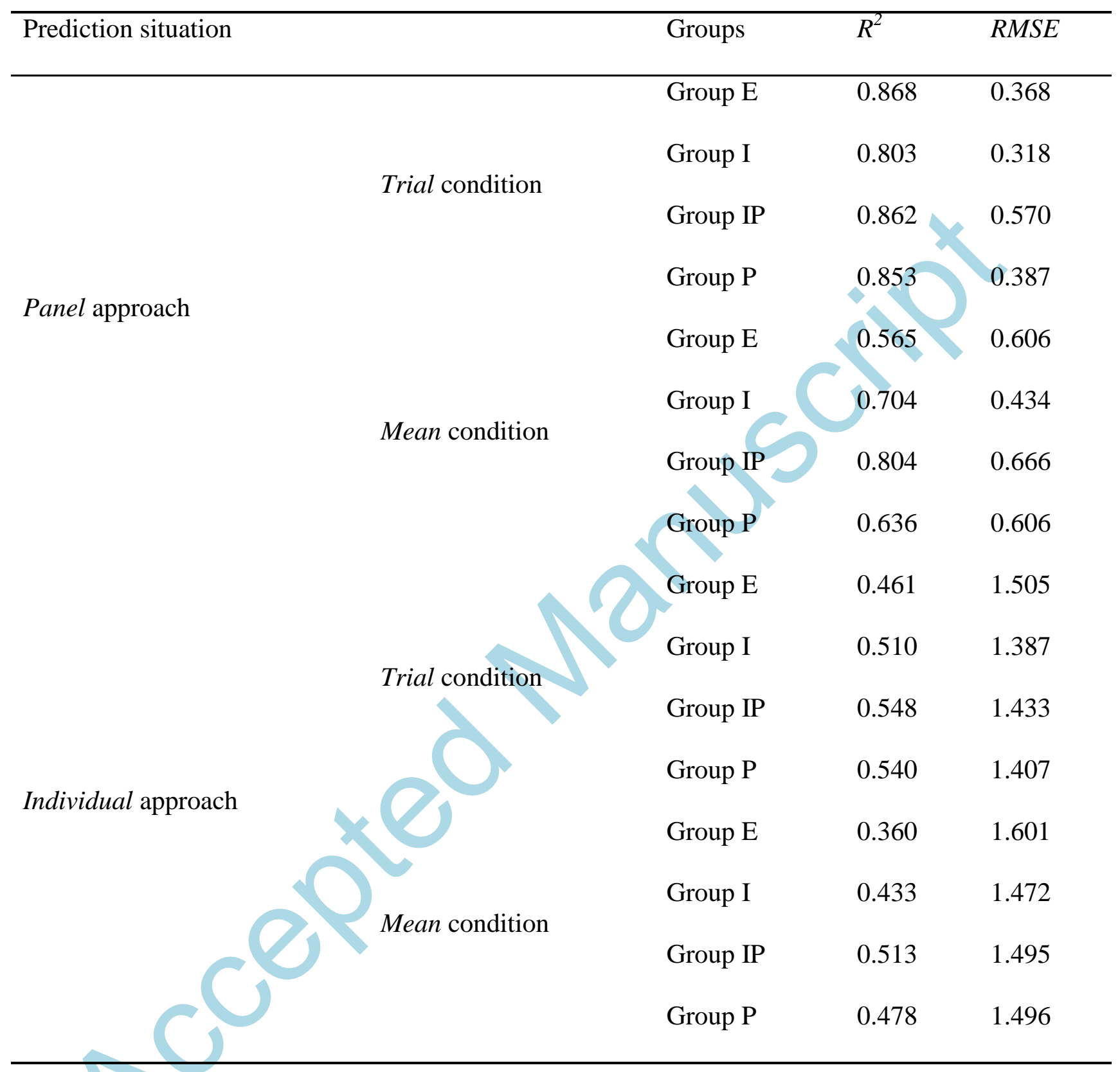




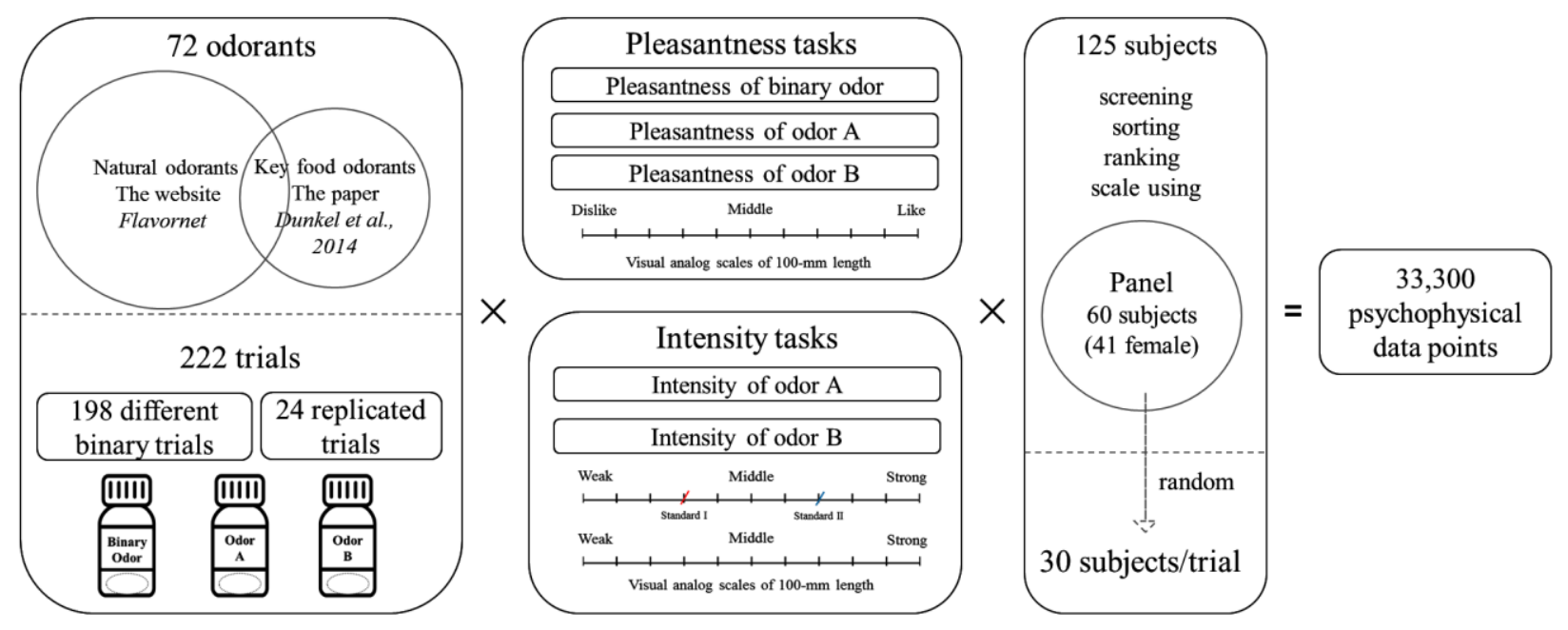


Odorants variance

Type Intensity Pleasantness

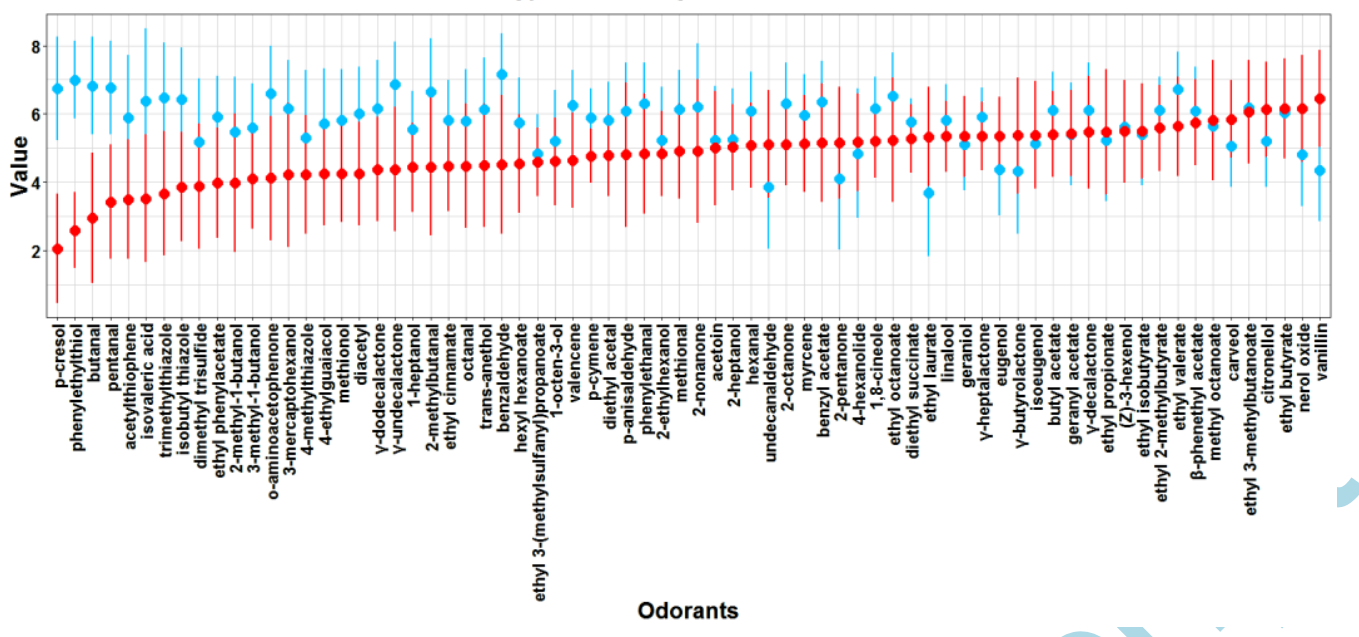

600 


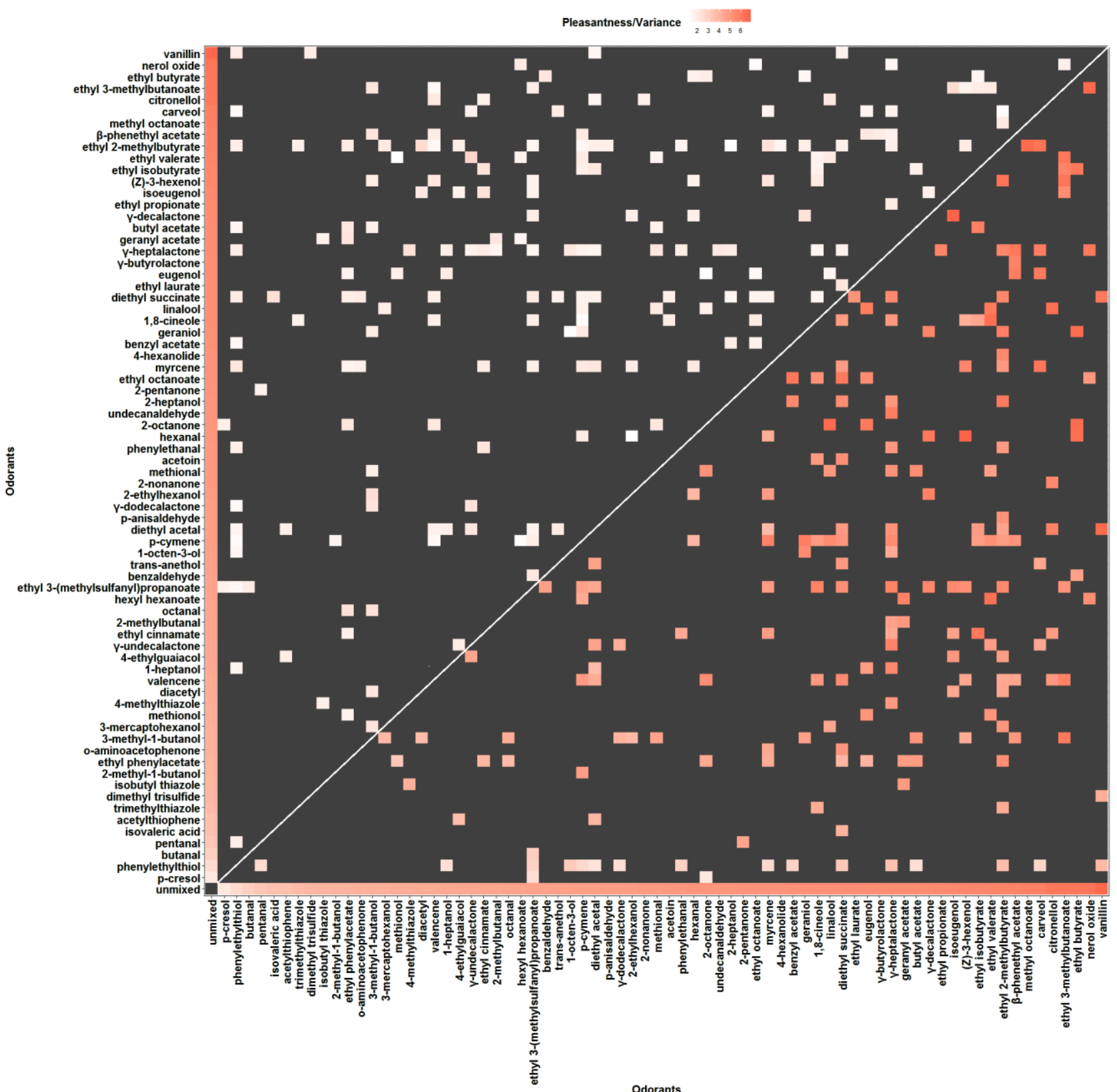

602

Odorants 
(a)

\section{Model $=$ Prediction from mean value $\triangleq$ Prediction from value in trial}

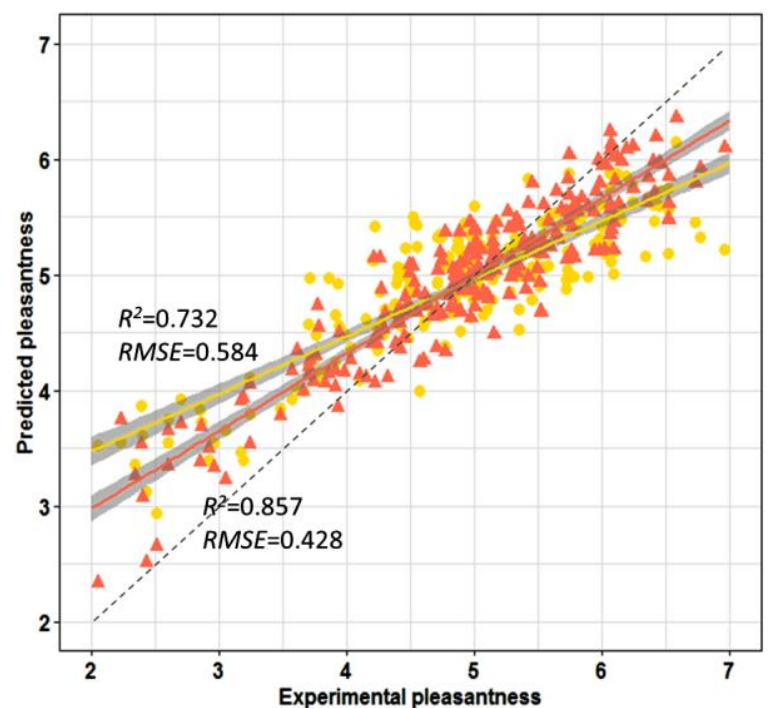

(b)

Model $\quad$ Prediction from mean value - Prediction from value in trial

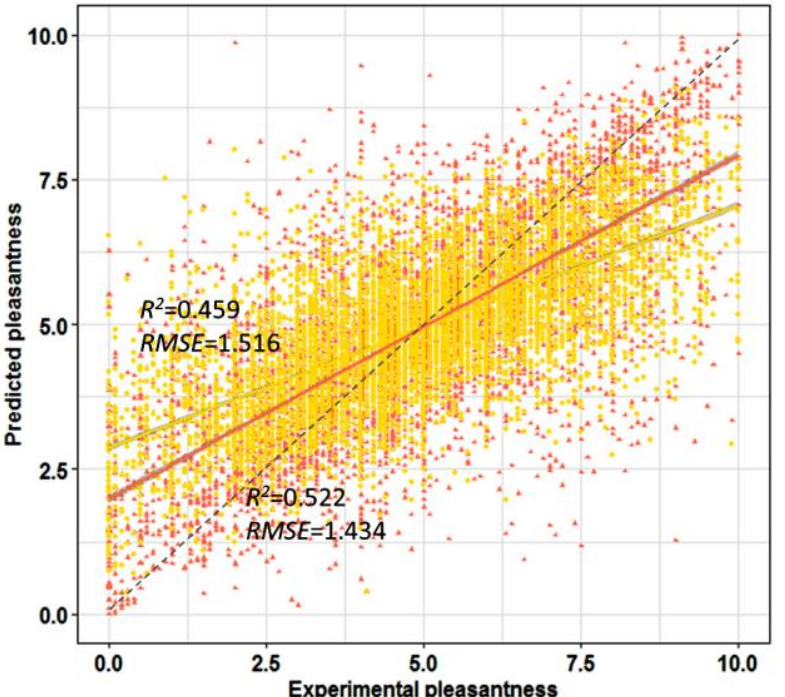

